\title{
Pregnancy Complication
}

National Cancer Institute

\section{Source}

National Cancer Institute. Pregnancy Complication. NCI Thesaurus. Code C34941.

The co-occurrence of pregnancy and a disease. The disease may precede or follow conception and it may or may not have a deleterious effect on the pregnant woman or fetus. 